\title{
Energy Efficiency Analysis of Effective Hydrocast for Underwater Communication
}

\author{
J. V. Anand and S. Titus \\ M.A.M College of Engineering, Trichy, Tamilnadu, India.
}

(Received 17 October 2014; accepted: 30 September 2015)

Underwater acoustic communication is difficult due to a low propagation speed, limited bandwidth, and energy consumption. There is a communication void primarily due to oceanic currents, which cause node mobility. Hence, protocol recovery has been focused on two means: Eulerian and Lagrangian. This work provides an analysis using the Eulerian approach with internetworking between sensors, wherein an effective hydrocast is proposed with the concept of triangulation to avoid long detour paths for recovery. The protocol Effective Hydrocast uses normalized advance wherethe cost is calculated with energy consumption, throughput, packet delivery ratio and propagation delay. Simulations with the ns 2 simulator using an aquasim patch with the development of an effective hydrocast protocol are done for throughput with two values of bandwidth minimal $3 \mathrm{kHz}$ and maximal $1000 \mathrm{kHz}$. The results of throughput are compared with depth based routing, energy efficient depth based routing, energy efficient fitness based routing, hydrocast and void aware pressure routing (VAPR) of which effective hydrocast is better. Then, values obtained for energy consumption between an effective hydrocast and VAPR obtained using ns 2 simulation is subjected to a time series analysis using a statistical package for social science. An autoregressive integrated moving average is developed and the best fitting model is estimated with the Bayesian information criterion, where an effective hydrocast is found to be better than VAPR.

\section{INTRODUCTION}

Acoustics in the underwater environment with sensor technology plays a vital role, as it distorts less than the electromagnetic and optical signals and spans for a longer distance. ${ }^{1}$ The propagation loss in an underwater environment is mainly characterized by geometrical spreading $(K)$ and attenuation. Geometrical spreading associates to the spreading of sound energy caused by the expansion of the wave fronts. The energy decomposition rate for spreading is characterized by transmission range $(R)$ from a source and it decomposes at a rate of $R^{-2}$ for spherical spreading and $R^{-1}$ for cylindrical spreading. ${ }^{1}$ The value of geometrical spreading is taken as $K=2$ (spherical spreading) for deep water and $K=1$ (cylindrical spreading) for shallow water, and $K=1.5$ for practical purposes, which was standardized. ${ }^{2}$

To overcome the communication void due to mobility and to find a proper recovery with minimal detour path, effective hydrocast was proposed. To forecast the protocol performance with future prediction in an uncertain underwater environment where data was obtained with simulation could be done using auto regressive integrated moving average. Hence, primary simulations were done in the ns 2 simulator with an aquasim patch $^{3}$ using the meandering current mobility model with the random waypoint model with various protocols, such as the depth based routing, ${ }^{4}$ energy efficient depth based routing, ${ }^{5}$ energy efficient fitness based routing, ${ }^{6}$ hydrocast $^{7}$ and void aware pressure routing (VAPR $)^{8}$ for throughput with minimal bandwidth of $3 \mathrm{kHz}$ and maximal bandwidth value of $1000 \mathrm{kHz}$ with the proposed effective hydrocast. Then, comparisons were made for energy consumption between VAPR and the effective hydrocast.

\section{NETWORK MODELS AND DEFINITIONS}

\subsection{Architectural Overview}

Two-dimensional architecture uses a group of sensor nodes to measure the ocean bottom, while the three-dimensional architecture was used to measure the ocean column at a preset depth. This paves the way for sensing coverage and communication coverage in a wireless scenario. ${ }^{2,9}$

\subsection{Underwater Protocols Based On Localization}

The taxonomy of routing protocols based on localisation in underwater acoustic sensor networks, which are classified as location-based and/or location-free. ${ }^{10}$ Location-based (geographical) routing in the location-based category assumes that each node knows some geographical information about itself and sinks to geographically identify the suitable progress area towards the sink. ${ }^{10}$ Location-free (non-geographical) routing protocols do not use full geographical information for routing. This can be classified as pressure-based routing (with a preset depth) and beacon-based routing. ${ }^{10}$ In the case of pressurebased routing protocols, every node is equipped with a depth sensor, which is made to float at a preset depth. ${ }^{10}$ The beaconbased approach uses ranging based on time of flight, angle of arrival, and round trip time. The beacon-approach can be stated as a periodic approach wherein beacon carries the necessary information. In a beacon-approach, network-wide synchronization is achieved with all sensor and anchor nodes, and a packet from the anchor determines its position. The number of anchors needed is three for the two dimensional geometry and four for the three dimensional geometry. ${ }^{11}$

\subsection{Disruption Tolerant Networks}

Connectivity and its void in connectivity due to node movement can be understood by using disruption tolerant networks (DTN). The underwater DTN uses two mechanisms for routing, namely scheduled contact and unscheduled contact mechanisms. ${ }^{12}$ A scheduled contact mechanism uses a base station, where concurrence must be obtained by two communicating sensor nodes with indirect links. ${ }^{12}$ Due to time varying channel conditions, this approach fails. An unscheduled contact mechanism does not use a base station and is suitable for time varying channels that exhibit irregularity. ${ }^{12}$ It can be divided 


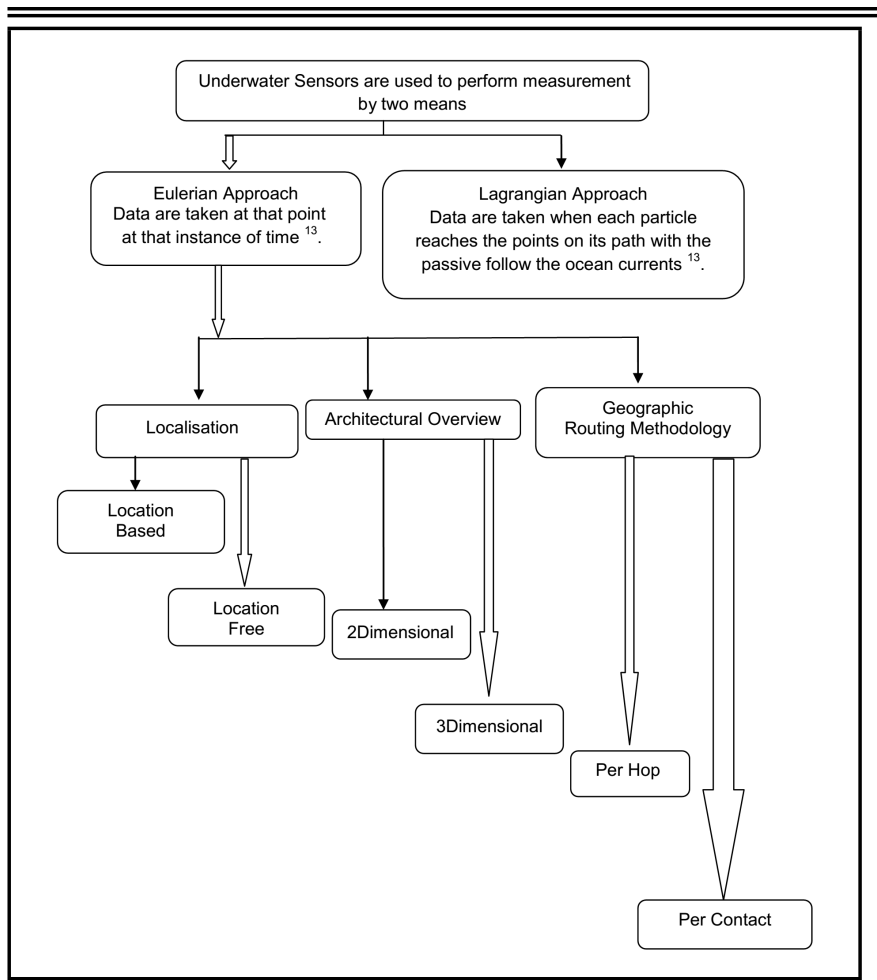

Figure 1. The work flow analysis. The designs in our simulations are depicted by broader arrows.

into opportunistic contact and predicated contact. An opportunistic contact, where a relay based scheme is in a node overhearing mode, can convey information by forwarding it to the destination. A predicted contact, on the other hand, is based on a simple mode where previous observations are taken into consideration, where no relay entities are used and where a direct mode of communication is deployed. ${ }^{12}$

\subsection{Geographic Routing}

Geographic routing or position-based routing uses location information for packet delivery in multi-hop wireless networks. It can be classified into per-contact routing and perhop routing. ${ }^{13}$ In per-contact routing, a node receiving data to be forwarded will exchange control packets and then select a suitable neighbouring node to forward the data to it, i.e., a neighbouring node with the minimal distance to the sink. The disadvantage of this protocol is high control overhead in percontact routing. ${ }^{13}$ In per-hop routing, the relaying nodes are determined by themselves. The source node or relaying nodes broadcast the data. The neighbouring node receiving the data will determine whether it is suitable to relay the data. The qualified nodes will be the relay nodes, and they will continue forwarding the data. The advantages of per-hop routing are its low control overhead and its fast forwarding, because no control packet exchange is incurred. ${ }^{13}$ Initially, the effective hydrocast uses per-contact routing and when there is a void the node uses per-hop routing for forwarding.

\section{SURVEY OF NETWORK PROTOCOLS WITH ITS SCHEMATIC ANALYSIS}

\subsection{Protocols for Void Avoidance with Recovery Using the Partially Lagrangian Approach}

Improved Adaptive mobility of courier nodes in Threshold Optimized DBR protocol (iAMCTD) is based on depth, residual energy, the signal-to-noise-ratio, and the sojourn tour of courier nodes. The selection of optimal forwarders is decided on the basis of the prioritization of signal quality index (a value based on local signal-to-noise-ratio), an energy cost function (based on enduring energy), and a depth dependent function (based on both local signal-to-noise-ratio and enduring energy). ${ }^{14}$ The void recovery paradigm with mechanical devices such as a floating buoy, autonomous underwater vehicles as in QUO VADIS, ${ }^{15}$ and Depth Controlled routing ${ }^{16}$ are not preferred due to the extra energy cost incurred by it.

\subsection{Protocols for Analyses without Void Avoidance using the Eulerian Approach}

In depth based routing (DBR) ${ }^{4}$ the sender forwards the packet to a set node at a lower depth than itself. Before it forwards the packet, it checks its packet history buffer to avoid frequent retransmission. If the packet is already in its packet history buffer, it checks its hop count. If it is less than the previous hop count, then it forwards the packet by adding in the priority queue. Otherwise, it discards the packet. It also has a timer that expires if it cannot find any neighbours within its transmission range. ${ }^{4}$ The problem with DBR is its increased number of packet transmissions and voids due to node mobility, where the sensor nodes are out of the transmission range. The depth-based multi-hop routing protocol ${ }^{17}$ for an underwater sensor network forwards packets using the depth and sleep wake algorithm. The sender forwards the packet to the place where only one node is selected as the next hop neighbour. Other nodes are in a sleep state. Only when they have a wake up control packet that makes them wake up and receive data. Otherwise, they continue in their sleep state. ${ }^{17}$ Energyefficient localization-free routing (EEDBR) ${ }^{5}$ forwards packets with depth and residual energy. It is similar to DBR, but it is sender based because the nodes on the record are sorted based on their remaining energy, which shows their precedence of forwarding. To prevent redundant data packet forwarding, each contender node judges a holding time according to its remaining energy and precedence, in which a smaller holding time is assigned to a node with more remaining energy and it receives the packet. ${ }^{5}$ The energy-efficient fitness (EEF) ${ }^{6}$ protocol which uses depth, residual energy, and fitness, which is calculated based on the distance between the sending node, forwarding node, and sink. ${ }^{6}$ Channel aware pressure routing $(\mathrm{CARP})^{18}$ is a stateful approach, which depends on number of hops, link quality, and energy consumption. Its methodology was based on contention based approach with handshaking methodology and used as PING PONG messages, where a shorter hop is preferred for the highest throughput, whereas longer hops are present for better link quality. ${ }^{18}$

\subsection{Protocols for Void Avoidance with Recovery and Message Based Transmission}

The message-based wireless protocol with geographic routing mostly uses normalized advance ${ }^{19}$ and considers the criteria of advance, which prefers a node that is closer to the sink and the furthest within its transmission range. Hydrocast, which uses a permissible delay and normalized advance where the cost is calculated by the packet delivery, does not provide energy efficiency and incurs long detour paths with void avoidance. ${ }^{7}$ Vector-based void avoidance uses concave and convex routing to overcome voids due to unequal load distribution among sensor nodes. However, this context depends upon the voids due to collisions. ${ }^{20}$ 


\subsection{Void Aware Pressure Routing}

Methodology of VAPR is as follows: ${ }^{8}$

1) Every sonobuoy on surface sends a beacon message.

2) Sensor that receives the beacon message updates its depth only if the beacon sequence is greater than the previous beacon sequence.

3) If the beacon sequence is equal to the previous beacon sequence, then it checks for its hop count.

4) If steps 2 and 3 are true, then it updates its next hop data forwarding direction (N_DF_Dir) and data forwarding direction (DF_Dir).

5) The direction for data flow is set 'up' when the beacon is received from shallower depth node otherwise it is set as 'down.'

6) Route Initialization is used to initialize each node's internal states:

i. Initially, each node in the network begins as an isolated local maximum with a hop count equal to infinity, except the sonobuoy.

ii. Then, it will have a connected component to one of the sonobuoy whose state will be changed to nonlocal maxima.

iii. If current sensor node is a source node, then set the data forwarding direction, hop count and sequence number as zero.

If the sensor node is a forwarding node, then set the data forwarding direction, next hop data forwarding direction, sequence number and the number of hops as infinity.

7) Data Forwarding: Nodes forward data solely based on the data forwarding direction (DF_Dir) and next hop data forwarding direction (N_DF_Dir).

8) Initialize all nodes in the connected set:

If the neighbour node depth is lesser than or equal to the present node depth then the data forwarding direction is equal to next hop data forwarding direction. This indicates the packets are transferred towards the sonobuoy which is indicated as downwards.

If the neighbour node depth is greater than the present node depth then the data forwarding direction is equal to next hop data forwarding direction. This indicates the packets are not transferred towards the sonobuoy. It should then transmit in the direction which is indicated as upwards.

9) Clustering:

i. Form all nodes in the forwarding set $(F)$ and select the best one.

ii. Get one hop neighbour from the best node and form cluster.

iii. Remove the best node from its one hop neighbour and from forwarding set.

iv. If $F$ is not empty, repeat steps i-iii, else Stop.
The purpose of the VAPR protocol discussed in detail is compared with the effective hydrocast. The problem with VAPR is that it is fully based on control messages, which increase the delay and overhead.

The protocols discussed in the literature either focus on greedy routing with energy consumption or greedy routing with a recovery mode. To the best of our knowledge, no protocol focuses on greedy routing with energy consumption, minimal overhead, and a minimal detour path so much so this work focuses on these constraints. The prior work of effective hydrocast ${ }^{21}$ is based on geographic routing with normalized advance, which is an advance divide by cost, wherein the cost was calculated with throughput, packet delivery ratio, energy consumption, and propagation delay was estimated for offered load (data packet generated by source node with energy consumption and throughput) with analytical analysis using regression. However, regression was found without lags and hence it fails to consider for the relationship through time and overestimates the relationship between the dependent and independent variables. So this work focuses on data obtained for time interval and comparisons with ARIMA model for forecasting.

\section{ALGORITHM DEVELOPMENT}

\subsection{Methodology}

All nodes initialize its surface ID and depth as infinity. Other than sonobuoy, all sensor nodes send and receive 'hello' messages periodically and update their neighbour information of their expected neighbour list. The source node then checks for the nodes within its upper hemispherical transmission range and computes the angle between neighbours using:

$$
\text { Hemisphere Transmission Range }=\frac{2 \times \pi^{2}}{(\text { Angle })^{2}} .
$$

Then, the verification is done to check whether the node reaches a local maxima point. If the nodes do not reach points of local maxima, then they can form a dominating triangle. The sender sends a message for triangle formulation along with the depth. Receiving nodes include that information in the triangle table maintained by each node with $x, y, z$ positions. Every node maintains its position, which is checked every 1 second. Table is formed with the three nodes information X1, X2, X3 and $\mathrm{X} 0$ which is the geographical centre of the triangle. Nodes then attach its depth, Data Forwarding direction, NADV (Normalized advance) Status

$$
\text { Normalized Advance }=\frac{\text { Advance }}{\text { Cost }} .
$$

Advance is the same criteria used in geographic routing and the cost is estimated using throughput with varying bandwidth, packet delivery ratio, energy consumption, and propagation delay. The formulas for estimating cost are:

$$
\text { Throughput }=\frac{\text { Received Packets } \times \text { Maximum Packet Size } \times 8}{\text { Current Time } \times \text { Bandwidth }} ;
$$

Packet Delivery Ratio $=\frac{\text { Received Packets }}{\text { Send Packets }} \times 100$;

Energy Consumption $=$ Initial Energy - Nodes Current Energy;

Propagation Delay $=\frac{\left(T_{2}-T_{1}\right)+\left(T_{4}-T_{3}\right)}{2} ;$ 


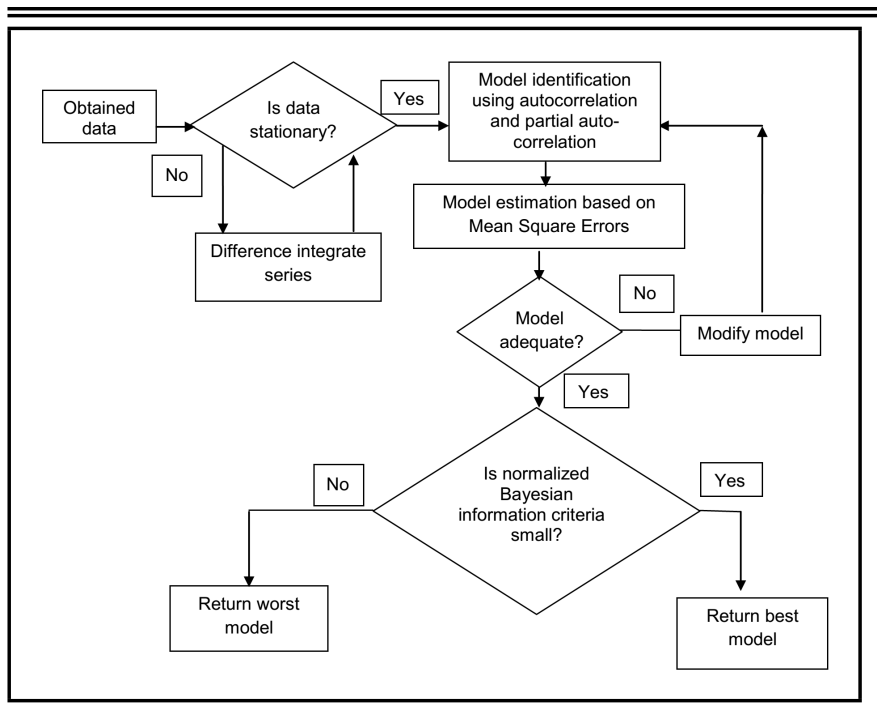

Figure 2. The flowchart for data analysis used in ARIMA model identification.

$T_{1}$ is the time stamp at sender and $T_{2}$ is the time stamp at receiver, $T_{3}$ is the time stamp of retransmission time at receiver and $T_{4}$ is the time stamp of reception time at the sender. So when a node comes under a communication void, then it knows the recovery nodes in its dominating triangle formulations thus avoiding long detour paths for recovery. In addition, if the node recovers from a void, then it switches back to the greedy mode. Otherwise, it tries to route towards a node within its communication capabilities, even in its lower hemispherical transmission range.

\section{DATA ANALYSIS}

\subsection{Time Series Model}

There are two methods to analyse the time series either in frequency domain (used for periodic and cyclical observations) or in time domain (estimates relationship using data collected over time). ${ }^{22}$ This work focuses on univariate autoregressive integrated moving average (ARIMA) model for data obtained in time domain for two protocols: the effective hydrocast and the void aware pressure routing.

\subsection{Autoregressive (AR) (Regression on Self)}

The value of the variable in one period is related to the value of the variable in the previous period. ${ }^{23} \mathrm{AR}(p)$ is an autoregressive model with

$$
y_{t}=a+\sum_{i=1}^{p} \psi_{i} \psi_{(t-i)}+\varepsilon_{t}
$$

where $a$ is a constant, $\psi_{i}$ is the coefficient of the model, $\psi_{(t-i)}$ is the lagged variable in time, and $\dot{\varepsilon}_{t}$ is the error (assumed to be normally distributed as well as independent error). The summation is taken till an order of polynomial $p$.

When regression is done there are errors (residuals) which have a time series structure. Adjusting the estimated regression coefficients and the errors are possible when it has time series structure and is called regression on self or auto-regressive function.

\subsection{Moving Average (MA)}

This model states for the option of a relationship between a variable and the residuals from previous model. ${ }^{23} \mathrm{MA}(q)$

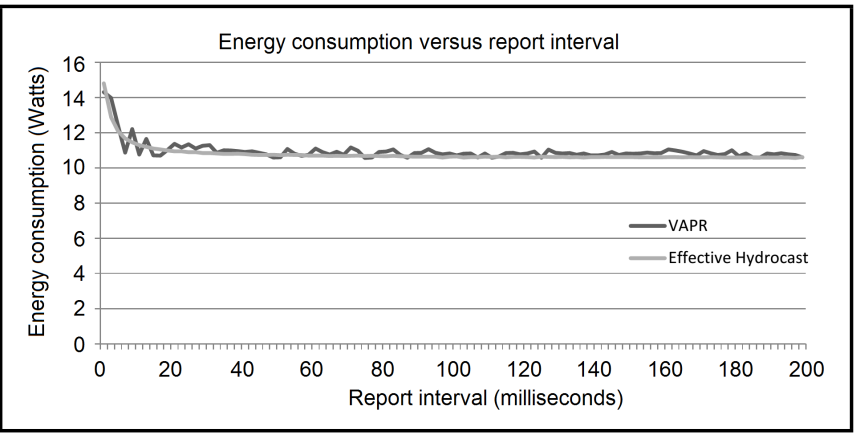

Figure 3. This energy consumption analysis was done to report the interval at two milliseconds, each till 200 milliseconds for 100 values for the effective hydrocast and void aware pressure routing, of which the effective hydrocast was found better than the VAPR protocol.

model with $q$ lags is:

$$
y_{t}=a+\dot{\varepsilon}_{t}+\sum_{i=1}^{q} \theta_{i} \dot{\varepsilon}_{(t-i)}
$$

where $\theta_{i}$ is the coefficient of the lagged error term in time $t-i$. The parameters of errors are not known and the optimal values have to be chosen by iterations until sum of squares of errors is minimized and average error is zero.

\subsection{Autoregressive Moving Average (ARMA)}

It combines $p$-th order autoregressive and $q$-th order moving average models, so it is called auto regressive moving average $(\operatorname{ARMA}(p, q)):^{22,24}$

$$
y_{t}=a+\sum_{i=1}^{p} \psi_{i} \psi_{(t-i)}+\dot{\varepsilon}_{t}+\sum_{i=1}^{q} \theta_{i} \dot{\varepsilon}_{(t-i)} .
$$

\subsection{Stationary (Standing in Time)}

Modelling an $\operatorname{ARMA}(p, q)$ process requires stationarity. The sequence of process, which is said to be random not only in time, but also in space, is called as an stationary process, if it has a mean and variance that do not change over time. If it is not stationary, the model is subjected to differencing and integrating the variables to develop an autoregressive integrated moving average (ARIMA). ARIMA $(p, d, q)$ denotes a ARMA model with $p$ autoregressive lags and $q$ moving average lags supplemented with $d$ differencing operations. ${ }^{24}$ Differencing here computes the difference among a pair of data at some time interval.

\subsection{Autocorrelation Function}

Autocorrelation function (ACF) is the proportion of the autocovariance of $y_{t}$ and $y_{t-k}$ to the variance of $y_{t}$ :

$$
\operatorname{ACF}(k)=\frac{\operatorname{COVARIANCE}\left(y_{t}, y_{t-k}\right)}{\operatorname{VARIANCE}\left(y_{t}\right)} .
$$

ACF gives the cross correlation between $y_{t}$ and $y_{t-k} \cdot{ }^{24,25}$

\subsection{Partial Autocorrelation Function}

Partial autocorrelation function (PACF) is the simple correlation between $y_{t}$ and $y_{(t-k)}$, where $y_{(t-k)}$ denotes the part explained by the intervening lags. ${ }^{25}$ PACF gives the amount of correlation between the variable and its lower order lags. 


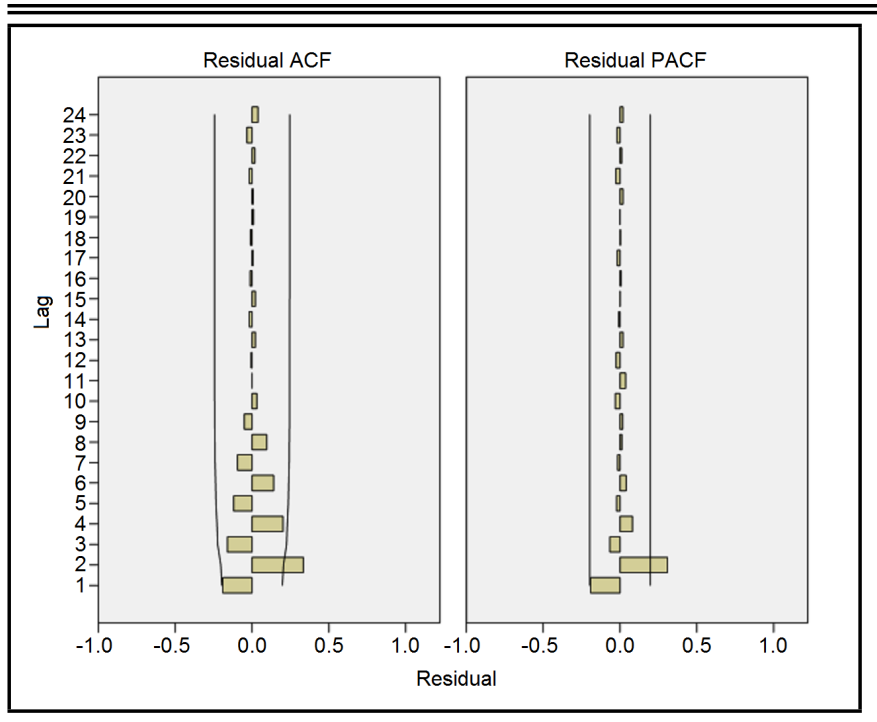

Figure 4. The autocorrelation function and partial autocorrelation function plot for graphing the residual of 24 lags for an effective hydrocast.

\subsection{Goodness of Fit Bayesian Information Criterion (BIC)}

In statistics, the Bayesian information criterion $(\mathrm{BIC})^{24,26}$ or Schwarz criterion (also SBC, SBIC) is a principle for model selection among a finite set of models. It is based upon the likelihood function and it is closely related to the Akaike information criterion (AIC). ${ }^{24,26}$ They measure the trade-off between the model fit and complexity of the model:

$$
\begin{aligned}
& \mathrm{AIC}=-2 \ln (L)+2 K ; \\
& \mathrm{BIC}=-2 \ln (L)+\ln (N) K ;
\end{aligned}
$$

where $L$ is the parameter for the likelihood functions assessed as parameter estimates, $N$ is the number of observations, and $K$ is the number of estimated parameters.

\subsection{Normalized Bayesian Information Crite- rion (Normalized BIC)}

Normalized BIC is a calculation of the overall fit of a model that attempts to report for model complexity. It is a score based upon the mean square error and includes a penalty for the number of parameters in the model and the length of the series. This consequence takes away the advantage of models with more parameters, making the statistic easy to compare across diverse models of the same series. ${ }^{26}$

\section{RESULTS AND DISCUSSION}

The simulation in our scenario are based on ns2 with aquasim patch $^{3}$ which incorporates the metrics of underwater using uw-common, uw-mac, uw-routing, and uw-tcl. ${ }^{3}$ The energy consumption on all the nodes was calculated by two means: first by calculating the residual energy of each node using Eq. (5) and secondly by calculating the overall energy consumption with the number of nodes:

$$
\text { Average Energy Consumption }=\frac{\text { Total Consumed Energy }}{\text { Number of nodes }} \text {. }
$$

Then, the series was analysed with the ARIMA model with the energy consumption as a dependent variable. For the void aware pressure routing the sequence of values was found to be stationary with ARIMA values of $(1,0,0)$. Then the effective hydrocast was found to be non stationary with ARIMA values $(0,1,1)$ after differentiation and integration.

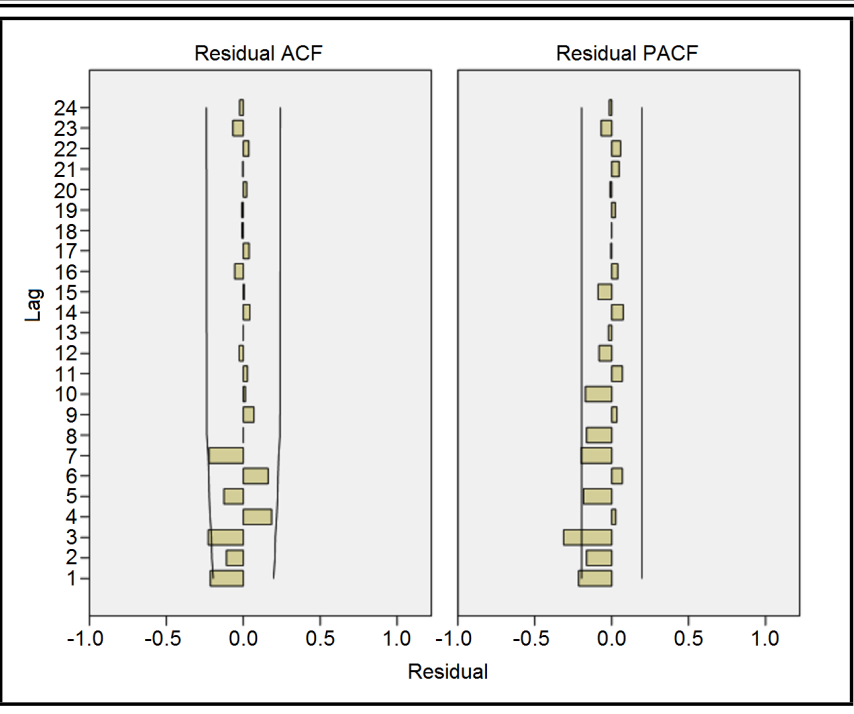

Figure 5. The autocorrelation function and partial autocorrelation function plot graphing of residual for 24 lags for void aware pressure routing.

\section{CONCLUSIONS}

In this paper, an effective hydrocast was proposed with a normalized advance where the criteria advance prefers the node that is closer to the sink and cost was estimated using packet delivery ratio, propagation delay, energy consumption, and throughput with minimal detour paths using triangulation. Then comparisons were made with depth-based routing, energy efficient depth based routing, energy efficient fitness based routing, hydrocast, and VAPR for throughput with minimal bandwidth of $3 \mathrm{kHz}$ and maximal bandwidth value of $1000 \mathrm{kHz}$. Comparisons of the effective hydrocast for energy consumption had been made with void aware pressure routing - a fully stateful approach for void avoidance, which uses beacon messages along with depth, direction, sequence number, and hop count mentored by a sonobuoy. It was analysed through simulations by using ns 2 based simulator with aquasim patch. The effective hydrocast with partially stateful approach was better with reduced control packet transmissions. Then, an autoregressive integrated moving average analysis had been developed for all the data obtained for energy consumption and the best model had been selected with minimal value of root mean square error and normalized Bayesian information criterion by using statistical package for social science. This analysis showed that the effective hydrocast was better than VAPR. Future work will deal with empirical characteristics of attenuation analysis in underwater with recovery from void using scalene triangle formulation.

\section{REFERENCES}

1 Preisig, J. Acoustic propagation considerations for underwater acoustic communications network development, ACM SIGMOBILE Mobile Computing and Communications Review, 11 (4), 2-10, (2007). http://dx.doi.org/10.1145/1347364.1347370

2 Akyildiz, I. F., Pompili, D., and Melodia, T. Underwater acoustic sensor networks: research challenges, Ad Hoc Networks, 3 (3), 257-279, (2005). http://dx.doi.org/10.1016/j.adhoc.2005.01.004

3 Xie, P., Zhou, Z., Peng, Z., Yan, H., Hu, T., Cui, J.-H., Shi, Z., Fei, Y., and Zhou, S. Aqua-Sim: An NS-2 based simulator for underwater sensor networks, Proceedings of 
J. V. Anand, et al.: ENERGY EFFICIENCY ANALYSIS OF EFFECTIVE HYDROCAST FOR UNDERWATER COMMUNICATION

Table 1. The values used for simulation parameters in our scenario.

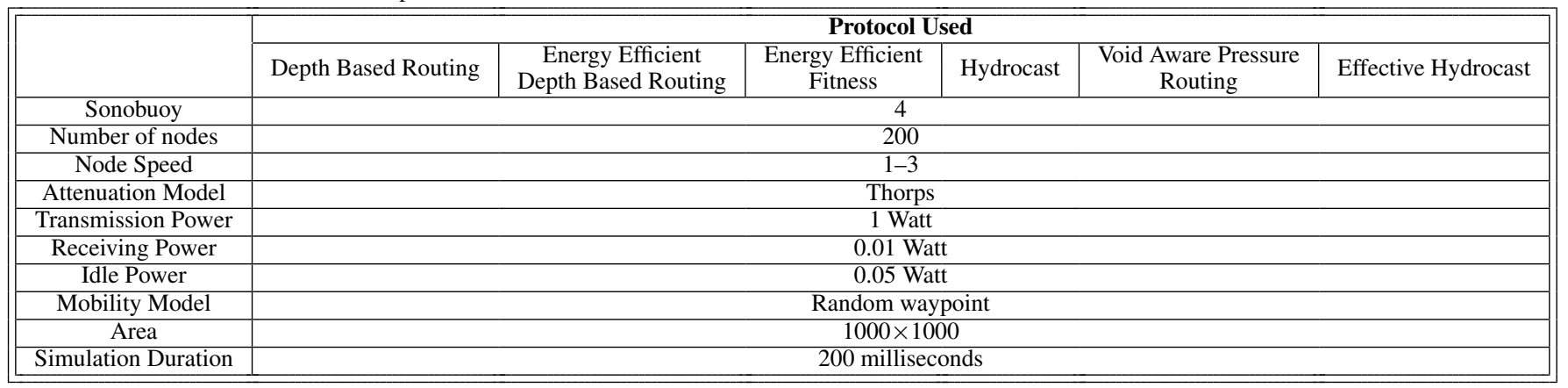

Table 2. The values used for estimating throughput in our scenario with a minimal of $3 \mathrm{kHz}$ used and maximum of $1000 \mathrm{kHz}$ within the acoustic range of which an effective hydrocast was better than all the other protocols.

\begin{tabular}{|c|c|c|c|c|c|c|}
\hline Bandwidth & Depth Based Routing & $\begin{array}{c}\text { Energy Efficient } \\
\text { Depth Based Routing }\end{array}$ & $\begin{array}{c}\text { Energy Efficient } \\
\text { Fitness }\end{array}$ & Hydrocast & $\begin{array}{c}\text { Void Aware Pressure } \\
\text { Routing }\end{array}$ & \begin{tabular}{c} 
Effective Hydrocast \\
\hline $3 \mathrm{kHz}$
\end{tabular} \\
\hline $1000 \mathrm{kHz}$ & 0.0000128 & 0.0000256 & 0.0000256 & 0.0018432 & 0.0024576 \\
0.0007168 & 0.0001536 & 0.0001536 & 0.1958400 & 0.1974270 \\
\hline
\end{tabular}

OCEANS 2009, MTS/IEEE Biloxi-Marine Technology for Our Future: Global and Local Challenges, 1-7, (2009).

4 Yan, H., Shi, Z. J., and Cui, J.-H. DBR: depth-based routing for underwater sensor networks, Proceedings of NETWORKING 2008 Ad Hoc and Sensor Networks, Wireless Networks, Next Generation Internet, 72-86, Springer, Berlin, Heidelberg, (2008). http://dx.doi.org/10.1007/9783-540-79549-0_7

5 Wahid, A. and Kim, D. An energy efficient localization-free routing protocol for underwater wireless sensor networks, International Journal of Distributed Sensor Networks, 8 (4), (2012). http://dx.doi.org/10.1155/2012/307246

6 Ashrafuddin, Md., Manowarul Islam, Md., and Mamun-orRashid, Md. Energy efficient fitness based routing protocol for underwater sensor network, International Journal of Intelligent Systems and Applications (IJISA), 5 (6), 61-69, (2013). http://dx.doi.org/10.5815/ijisa.2013.06.08

7 Lee, U., Wang, P., Noh, Y., Vieira, L. F. M., Gerla, M., and Cui, J.-H. Pressure routing for underwater sensor networks, Proceedings of IEEE INFOCOM 2010, 1-9, (2010). http://dx.doi.org/10.1109/INFCOM.2010.5461986

8 Noh, Y., Lee, U., Wang, P., Choi, B. S. C., and Gerla, M. VAPR: void-aware pressure routing for underwater sensor networks, IEEE Transactions on Mobile Computing, 12 (5), 895-908, (2013). http://dx.doi.org/10.1109/TMC.2012.53

9 Akyildiz, I. F., Pompili, D., and Melodia, T. Stateof-the-art in protocol research for underwater acoustic sensor networks, Proceedings of the 1st ACM International Workshop on Underwater Networks, 7-16, (2006). http://dx.doi.org/10.1145/1161039.1161043

10 Kheirabadi, M. T. and Mohamad, M. M. Greedy routing in underwater acoustic sensor networks: a survey, International Journal of Distributed Sensor Networks, 9 (7), (2013). http://dx.doi.org/10.1155/2013/701834

11 Ramezani, H. and Leus, G. Ranging in an underwater medium with multiple isogradient sound speed profile layers, Sensors, 12 (3), 2996-3017, (2012). http://dx.doi.org/10.3390/s120302996

12 Cho, H.-H., Chen, C.-Y., Shih, T. K., and Chao, H.-C. Survey on underwater delay/disruption tolerant wireless sensor network routing, IET Wireless Sensor Systems, 4 (3), 112121, (2014). http://dx.doi.org/10.1049/iet-wss.2013.0118

13 Chen, Y.-D., Lien, C.-Y., Wang, C.-H., and Shih, K.-P. DARP: A depth adaptive routing protocol for large-scale underwater acoustic sensor networks, Proceedings of OCEANS 2012 - Yeosu, 1-6, (2012). http://dx.doi.org/10.1109/OCEANS-Yeosu.2012.6263505

14 Javaid, N., Jafri, M. R., Khan, Z. A., Qasim, U., Alghamdi, T. A., and Ali, M. IAMCTD: Improved adaptive mobility of courier nodes in threshold-optimized dbr protocol for underwater wireless sensor networks, International Journal of Distributed Sensor Networks, 10 (11), (2014). http://dx.doi.org/10.1155/2014/213012

15 Chen, B. and Pompili, D. QUO VADIS: QoS-aware underwater optimization framework for inter-vehicle communication using acoustic directional transducers, Proceedings of the 8th Annual IEEE Communications Society Conference on Sensor, Mesh and Ad Hoc Communications and Networks (SECON), 341-349, (2011). http://dx.doi.org/10.1109/SAHCN.2011.5984916

16 Coutinho, R. W. L., Vieira, L. F. M., and Loureiro, A. DCR: Depth-Controlled routing protocol for underwater sensor networks, Proceedings of the IEEE Symposium on Computers and Communications (ISCC), 000453-000458, (2013). http://dx.doi.org/10.1109/ISCC.2013.6754988

17 Guangzhong, L. and Zhibin, L. Depth-based multi-hop routing protocol for underwater sensor network, Proceedings of the 2nd International Conference on Industrial Mechatronics and Automation (ICIMA), 2, 268-270, (2010). http://dx.doi.org/10.1109/ICINDMA.2010.5538317

18 Basagni, S., Petrioli, C., Petroccia, R., and Spaccini, D. Channel-aware routing for underwater wireless networks, Proceedings of OCEANS 2012 - Yeosu, 1-9, (2012). http://dx.doi.org/10.1109/OCEANS-Yeosu.2012.6263538

19 Lee, S., Bhattacharjee, B., and Banerjee, S. Efficient geographic routing in multihop wireless networks, Proceedings of the 6th ACM International Symposium On Mobile Ad Hoc Networking And Computing, 230-241, (2005). http://dx.doi.org/10.1145/1062689.1062720 
Table 3. The energy consumption obtained for a duration of 200 milliseconds.

\begin{tabular}{|c|c|c|}
\hline $\begin{array}{l}\text { Report Interval } \\
\text { (Milliseconds) }\end{array}$ & $\begin{array}{l}\text { Effective Hydrocast } \\
\text { (Watts) }\end{array}$ & $\begin{array}{c}\text { Void Aware Pressure Routing } \\
\text { (Watts) }\end{array}$ \\
\hline 2 & 14.8041 & 14.3095 \\
\hline 4 & 12.8916 & 13.9857 \\
\hline 6 & 12.0827 & 12.3933 \\
\hline 8 & 11.6686 & 10.8739 \\
\hline 10 & 11.4532 & 12.2012 \\
\hline 12 & 11.2997 & 10.7577 \\
\hline 14 & 11.1975 & 11.6511 \\
\hline 16 & 11.0979 & 10.7143 \\
\hline 18 & 11.0533 & 10.7006 \\
\hline 20 & 10.9957 & 11.0213 \\
\hline 22 & 10.9416 & 11.3662 \\
\hline 24 & 10.9471 & 11.1653 \\
\hline 26 & 10.8915 & 11.3429 \\
\hline 28 & 10.8900 & 11.0994 \\
\hline 30 & 10.8502 & 11.2544 \\
\hline 32 & 10.8416 & 11.2920 \\
\hline 34 & 10.8168 & 10.8549 \\
\hline 36 & 10.7922 & 11.0019 \\
\hline 38 & 10.7930 & 10.9884 \\
\hline 40 & 10.8080 & 10.9595 \\
\hline 42 & 10.7810 & 10.9096 \\
\hline 44 & 10.7426 & 10.9479 \\
\hline 46 & 10.7415 & 10.8599 \\
\hline 48 & 10.7406 & 10.7733 \\
\hline 50 & 10.7485 & 10.5962 \\
\hline 52 & 10.7287 & 10.6059 \\
\hline 54 & 10.7529 & 11.0722 \\
\hline 56 & 10.7338 & 10.8041 \\
\hline 58 & 10.7378 & 10.6838 \\
\hline 60 & 10.7018 & 10.7465 \\
\hline 62 & 10.7013 & 11.1037 \\
\hline 64 & 10.6970 & 10.8962 \\
\hline 66 & 10.6791 & 10.7597 \\
\hline 68 & 10.6902 & 10.9215 \\
\hline 70 & 10.6707 & 10.7448 \\
\hline 72 & 10.6850 & 11.1603 \\
\hline 74 & 10.6964 & 10.9839 \\
\hline 76 & 10.6764 & 10.5660 \\
\hline 78 & 10.6903 & 10.5900 \\
\hline 80 & 10.6710 & 10.9032 \\
\hline 82 & 10.6609 & 10.9323 \\
\hline 84 & 10.6832 & 11.0493 \\
\hline 86 & 10.6691 & 10.7379 \\
\hline 88 & 10.6455 & 10.5825 \\
\hline 90 & 10.6446 & 10.8489 \\
\hline 92 & 10.6420 & 10.8529 \\
\hline 94 & 10.6407 & 11.0639 \\
\hline 96 & 10.6408 & 10.8591 \\
\hline 98 & 10.5880 & 10.7734 \\
\hline 100 & 10.6433 & 10.8221 \\
\hline
\end{tabular}

\begin{tabular}{|c|c|c|}
\hline $\begin{array}{l}\text { Report Interval } \\
\text { (Milliseconds) }\end{array}$ & $\begin{array}{l}\text { Effective Hydrocast } \\
\text { (Watts) }\end{array}$ & $\begin{array}{l}\text { Void Aware Pressure Routing } \\
\text { (Watts) }\end{array}$ \\
\hline 102 & 10.6534 & 10.7115 \\
\hline 104 & 10.5951 & 10.8126 \\
\hline 106 & 10.6193 & 10.8245 \\
\hline 108 & 10.6156 & 10.5935 \\
\hline 110 & 10.6433 & 10.8262 \\
\hline 112 & 10.6312 & 10.5813 \\
\hline 114 & 10.6406 & 10.6502 \\
\hline 116 & 10.6036 & 10.8506 \\
\hline 118 & 10.6276 & 10.8639 \\
\hline 120 & 10.6214 & 10.7786 \\
\hline 122 & 10.6115 & 10.8173 \\
\hline 124 & 10.5950 & 10.9254 \\
\hline 126 & 10.6392 & 10.5818 \\
\hline 128 & 10.6306 & 11.0392 \\
\hline 130 & 10.6197 & 10.8570 \\
\hline 132 & 10.6214 & 10.8192 \\
\hline 134 & 10.6027 & 10.8405 \\
\hline 136 & 10.6182 & 10.7526 \\
\hline 138 & 10.5925 & 10.8264 \\
\hline 140 & 10.6147 & 10.7069 \\
\hline 142 & 10.6199 & 10.7122 \\
\hline 144 & 10.6229 & 10.7628 \\
\hline 146 & 10.6105 & 10.9044 \\
\hline 148 & 10.6119 & 10.7348 \\
\hline 150 & 10.6104 & 10.8190 \\
\hline 152 & 10.6151 & 10.8143 \\
\hline 154 & 10.5986 & 10.8228 \\
\hline 156 & 10.6013 & 10.8744 \\
\hline 158 & 10.6016 & 10.8334 \\
\hline 160 & 10.6035 & 10.8473 \\
\hline 162 & 10.6182 & 11.0584 \\
\hline 164 & 10.6151 & 10.9880 \\
\hline 166 & 10.6031 & 10.9155 \\
\hline 168 & 10.6172 & 10.8224 \\
\hline 170 & 10.6062 & 10.7149 \\
\hline 172 & 10.6018 & 10.9599 \\
\hline 174 & 10.6148 & 10.8326 \\
\hline 176 & 10.5976 & 10.7311 \\
\hline 178 & 10.5941 & 10.7808 \\
\hline 180 & 10.5951 & 11.0003 \\
\hline 182 & 10.5956 & 10.6626 \\
\hline 184 & 10.5959 & 10.8218 \\
\hline 186 & 10.5968 & 10.5945 \\
\hline 188 & 10.5944 & 10.5937 \\
\hline 190 & 10.5943 & 10.8174 \\
\hline 192 & 10.5947 & 10.7732 \\
\hline 194 & 10.5960 & 10.8359 \\
\hline 196 & 10.5958 & 10.7695 \\
\hline 198 & 10.5651 & 10.7361 \\
\hline 200 & 10.6119 & 10.6040 \\
\hline
\end{tabular}

Table 4. The values used for estimating the model using normalized Bayesian information criterion of which effective hydrocast was found to be better than VAPR.

\begin{tabular}{|c|c|c|c|c|c|c|}
\hline \multirow{2}{*}{ MODEL } & \multicolumn{3}{|c|}{ MODEL FIT STATISTIC } & \multicolumn{3}{|c|}{ Ljung box Q } \\
\cline { 2 - 7 } & Stationary R Square & RMSE & Normalized BIC & Statistics & DF & Sig \\
\hline EFECTIVE HYDROCAST & 0.015 & 0.211 & -3.063 & 28.385 & 17 & 0.041 \\
\hline VOID AWARE PRESSURE ROUTING & 0.245 & 0.468 & -1.427 & 26.524 & 17 & 0.065 \\
\hline
\end{tabular}

20 Xie, P., Zhou, Z., Peng, Z., Cui, J.-H., and Shi, Z. Void avoidance in three-dimensional mobile underwater sensor networks, Wireless Algorithms, Systems, and Applications, 305-314, Springer Berlin Heidelberg, (2009). http://dx.doi.org/10.1007/978-3-642-03417-6_30

21 Anand, J. V. and Titus., S. Regression based analysis of effective hydrocast in underwater environment, Proceedings of the IEEE Region 10 Conference TENCON 2014, 1-6, (2014). http://dx.doi.org/10.1109/TENCON.2014.7022394

22 Brockwell, P. J. and Davis, R. A. Time Series: Theory and Methods, Springer Science \& Business Media, 1-266, (2013).

23 Melard, G. Algorithm AS 197: A fast algorithm for the exact likelihood of autoregressive-moving average models, Applied Statistics, 33 (1), 104-114, (1984). http://dx.doi.org/10.2307/2347672

${ }^{24}$ Choi, B. S. ARMA Model Identification, Springer Science \& Business Media, 29-75, (2012).

25 Bartlett, M. S. On the theoretical specification and sampling properties of autocorrelated time-series, Supplement to the Journal of the Royal Statistical Society, 8 (1), 27-41, (1946). http://dx.doi.org/10.2307/2983611

26 Schwarz, G. Estimating the dimension of a model, The Annals of Statistics, 6 (2), 461-464, (1978). http://dx.doi.org/10.1214/aos/1176344136 